\title{
Performance and Equilibrium Analysis of Heterogeneous IEEE 802.11 Based WLANs
}

\author{
Hao Zhu \\ Telecommunication and Information Technology Institute \\ Florida International University \\ 10555 W. Flagler Street, Miami, FL 33174 \\ hao.zhu@fiu.edu
}

\begin{abstract}
This paper presents a general model to study the medium access control (MAC) layer performance and equilibrium of WLANs consisting of nodes with different MAC parameters (e.g., backoff window size). Our model can be used in general 802.11-based WLANs since it captures the important factors such as non-uniform backoff behavior, channel errors and unsaturated traffic. We first formalize the performance of 802.11 based MAC protocols with simultaneous fixed point equations. We then derive the average per-flow service time of each flow, and apply it to calculate throughput. More importantly, based on our model, we use interval analysis to formally study the existence and uniqueness of the network's equilibrium, and find the sufficient condition for the uniqueness of equilibrium. We validate our model through simulations and the simulation results show that the model is quite accurate.
\end{abstract}

\section{Introduction}

With the low cost and high data rates, IEEE 802.11 based wireless local area networks (WLANs) have been widely deployed in residences, hotels, hospitals and other public areas as a communication infrastructure for high speed wireless Internet access. The core technology of 802.11 WLANs follows the medium access control (MAC) and physical layer (PHY) specifications [1] finalized by IEEE 802.11 working groups. In these specifications, the building block of medium access control mechanism is called distributed coordination function (DCF). DCF is a random access scheme and relies on the carrier sense multiple access with collision avoidance (CSMA/CA). Binary exponential backoff mechanism is used for data retransmission upon a collision or transmission failure. IEEE 802.11 working group has proposed a family of 802.11 MAC protocols such as 802.11 b/g/e [12] to improve the system performance in different aspects (e.g. channel capacity, service differentiation). Therefore, it is expected that future WLANs may need to accommodate nodes running heterogeneous 802.11 based MAC protocols. In order to have deep insights into the performance of heterogeneous 802.11 based WLANs, it is critical to develop a general model for 802.11 based WLANs considering the coexistence of heterogeneous MAC parameters since 
they play important roles in determining the bandwidth share of each node and the bandwidth utilization of the network.

There have been a number of studies focusing on the performance model of DCF. Bianchi 3] modeled the binary exponential backoff under saturated traffic conditions as a two-dimensional discrete Markov Chain model. The similar Markovian technique has been subsequently adopted by many other works 4.5. in modeling WLANs under unsaturated traffic. Carvalho and Garcia-LunaAceves [6] modeled the packet service time in single-hop WLANs given that the state probabilities of a node's backoff operation are known. With the results from [3], the first two moments of the service time were studied. Kumar et al. [7] proposed a simpler model by viewing the backoff procedure as a renewal process. Ramaiyan et al. 8] refined such approach to study the stability of the network in 802.11e WLANs [2] under saturated traffic conditions and give sufficient conditions for the existence and uniqueness of equilibrium. Kim and Hou [9] modeled the service time in large-scale single-hop WLANs for fast simulations. Medepalli and Tobagi [10] applied the average cycle time analysis to model the average service time in wireless ad hoc networks. Their model captures the impacts of unsaturated traffic and channel errors among contending nodes. However, the above-mentioned works are not sufficient to model the performance of heterogeneous 802.11 based WLANs under both saturated and unsaturated traffic.

In this paper, we present a general model of heterogeneous 802.11 based WLANs. The model is fairly simple and quite accurate. Our work differs from previous works in at least one of the following three aspects (especially the third aspect): first, our model captures the performance of general 802.11 based WLANs since we take into account of channel errors and realistic network operating scenarios (e.g., unsaturated traffic). Second, our model can be used to study the performance of heterogeneous 802.11 WLANs in which each node may have different system parameters (e.g. back-off window size). Third, we theoretically study the sufficient condition for the uniqueness of equilibrium in a general heterogeneous 802.11 WLANs. Our sufficient condition is a superset of that in [8].

We first model the performance of the MAC protocol using the fixed point analysis [7] using renewal process theory [11. The time average performance of the network can be obtained by solving simultaneous non-linear equations. Then, we apply interval analysis [12] to study the sufficient condition for the uniqueness of root, which indicates the network will be in a unique equilibrium. In the previous study [7] for homogeneous 802.11 WLANs, it has been shown that the network has a unique equilibrium if the mean backoff window sizes at different backoff stages are a non-decreasing sequence. Through our analysis, we prove that this sufficient condition still holds for a general heterogeneous 802.11 WLAN. We verify our model through simulations and the results show that our model is able to accurately capture the average behavior of the network.

The rest of the paper is organized as follows. Section 2 states the system model. Section 3 describes the details of the proposed analytical model. We evaluate our model in Section 4. Section 5 concludes this paper. 


\section{System Model}

We study a single-cell WLAN with fixed number $n$ of contending nodes that access the same wireless channel following 802.11 DCF [1. For simplicity, these nodes are within the transmission range of each other, and there is not hidden terminal problem. We consider general traffic situation for each node. In particular, node $i$ can send a packet to any other nodes in the network, and its aggregate traffic rate is $\lambda_{i}$ (in packet per slot). In order to capture the impact of unsaturated traffic sources, we assume that each node has the probability that its queue is non-empty, denoted by $\rho_{i}$. Suppose the average service time for a packet once it becomes the head of the queue is $E\left(S_{i}\right)$ (in slots), according to the Little's Theorem [11, we have

$$
\rho_{i}=\lambda_{i} E\left(S_{i}\right)
$$

Queueing theory [1] has shown that the average packet delay of node $i$ exponentially increases with $\rho_{i}$. According to delay requirements, we assume node $i$ operates with a targeted $\rho_{i}$ by controlling $\lambda_{i}$ based on Eq.(11). The control is deterministic if $E\left(S_{i}\right)$ can be uniquely determined by $\left(\rho_{1}, \ldots, \rho_{n}\right)$. We will prove that this assumption actually holds. According to the traffic and channel condition statistics, we assume the mean bit error rate of node $i$ is $b_{e} r_{i}$ and data packets sent by node $i$ have a mean length of $D A T A_{i}$. We assume the RTS/CTS handshaking mechanism is always switched on. As shown in [39, there is not significant differences in modeling these two mechanisms.

We also consider heterogeneous MAC parameters of each node. Even though the results in [8] can be used to model the impact of arbitrary inter frame space (AIFS) [2], it has been indicated in [8] that differentiating AIFS or the exponential component $p$ would starve low priority traffic as the load of system increases. On the contrary, using initial back-off window can produce a bounded throughput ratio between different classes of traffic. Thus, we simply focus on the case that the nodes may have heterogeneous backoff parameters. Specifically, backoff parameters (for node $i$ ) are:

- $K_{i}$ : At the $\left(K_{i}+1\right)$ th attempt either the impending packet of node $i$ succeeds or is discarded

$-b_{i, k}$ : The mean backoff (in slots) at the $k$ th attempt for an impending packet of node $i$, where $0 \leq k \leq K_{i}$.

Other parameters not mentioned are the same for all nodes. Following the design principle of standard IEEE 802.11 DCF [1], we assume that $b_{i, k}, k \geq 0$ is a nondecreasing sequence.

\section{The Proposed Model}

In this section, we first introduce the proposed model and then study the network's equilibrium. To make the presentation clear, we omit the inter frame spaces since their are much shorter than the transmission time of control and data packets. 


\subsection{Modeling the Attempt Rate}

Suppose node $i$ sends a data packet $D A T A_{i}$ with ber $_{i}$, the probability of transmission failures, denoted by $p_{e, i}$, can be calculated as:

$$
p_{e, i}=1-\left(1-\operatorname{per}_{i}(R T S)\right)\left(1-\operatorname{per}_{i}(C T S)\right)\left(1-\operatorname{per}_{i}\left(D A T A_{i}\right)\right)\left(1-\operatorname{per}_{i}(A C K)\right)
$$

where the packet error probability $\operatorname{per}_{i}(p)$ is defined by:

$$
\operatorname{per}_{i}(p)=1-\left(1-\text { ber }_{i}\right)^{\text {p.length }}
$$

When a transmission failure happens at node $i$, the amount of bandwidth waste, denoted by $\alpha_{i}$ can be calculated as:

$$
\alpha_{i}=t x(R T S)+t x(C T S)+\left(1-c_{i}\right)\left(t x\left(D A T A_{i}\right)+t x(A C K)\right)
$$

where $\operatorname{tx}(\mathrm{p})$ is the total transmission time of packet $p$ and

$$
c_{i}=\frac{\left(\operatorname{per}_{i}(R T S)+\operatorname{per}_{i}(C T S)\right)}{p_{e, i}}
$$

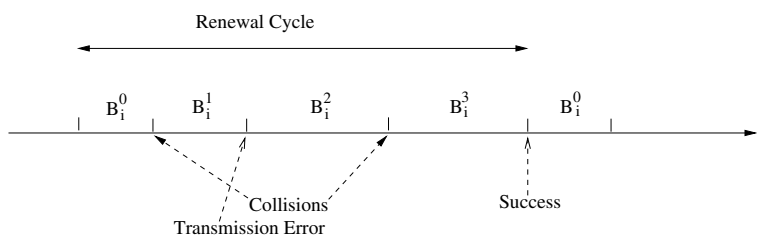

Fig. 1. The aggregate attempt process of node $i$

Whenever the channel is occupied due to either data transmissions or collisions, each node freezes its backoff timer until the channel is available. Therefore, similar to [7, we can remove all busy periods of the channel and study the attempt rate of each node according to the aggregate attempt process in the idle periods of the channel. As shown in Figure 1, the aggregate attempt process of a node can be seen as a renewal process in which the reward is the number attempts in each renewal cycle. Since the each node enters the next backoff stage when it has a collision or transmission failure, suppose the conditional collision probability seen by node $i$ when it attempts to access channel is $p_{c, i}$, the conditional probability that an attempt of the node fails, denoted by $\gamma_{i}$ follows:

$$
\gamma_{i}=\left(1-p_{e, i}\right) p_{c, i}+p_{e, i}
$$

With the decoupling assumption introduced by Bianchi $[3$ and the renewal reward theorem, the attempt rate of node $i$, denoted by $\beta_{i}$, is given by:

$$
\beta_{i}=\frac{\sum_{j=0}^{K_{i}} \gamma_{i}^{j}}{\sum_{j=0}^{K_{i}} b_{i, k} \gamma_{i}^{j}}
$$

where $b_{i, k}$ is the mean $k^{t h}$ backoff period of node $i$. 


\subsection{Modeling the Service Time}

Before studying the existence and uniqueness of equilibrium, we first model the service time of node $i$, denoted by $E\left(S_{i}\right)$. Equation (7) only describes the saturation attempt probability. Given the non-empty queue probability of node $i$, the actual attempt probability of node $i$ is equal to $\rho_{i} \beta_{i}$. Thus the conditional collision probability of node $i$ can be calculated by:

$$
p_{c, i}=1-\prod_{j=1, j \neq i}^{n}\left(1-\rho_{j} \beta_{j}\right)
$$

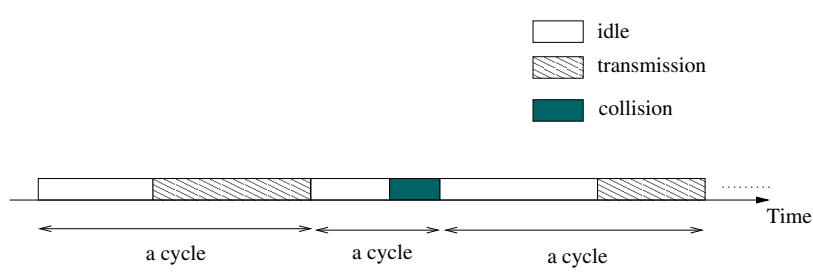

Fig. 2. The Illustration of Channel Activities

Since, as shown in Figure 2, the aggregate channel activity can be viewed as a renewal process, each of which contains an idle period followed by a collision or a transmission. For node $i$, its average service time $E\left(S_{i}\right)$ is the average delay for a packet from the time it reaches the head of the queue at node $i$ to the time the packet is transmitted or dropped. For the aggregate channel activity, the mean renewal time is the mean channel idle time plus the mean busy time for a transmission or a collision. We then study $E\left(S_{i}\right)$ conditioned on node $i$ having a packet in its queue. In this situation, the probability that a slot is idle, denoted by $P_{i d l e}$, is

$$
P_{i d l e, i}=\left(1-\beta_{i}\right) \prod_{j=1, j \neq i}^{n}\left(1-\rho_{j} \beta_{j}\right)
$$

Thus, the aggregate attempt rate is geometrically distributed with parameter $1-P_{i d l e, i}$, thus the mean channel idle time seen by node $i$ follows (Note that we use a slot as time unit):

$$
E\left(t_{i d l e, i}\right)=\frac{1}{1-P_{\text {idle }, i}}
$$

When the idle period ends, an attempt happens and the channel becomes busy due to a transmission or a collision. The probability of node $k$ performing an attempt seen by node $i$ follows:

$$
P_{a}(k, i)=\left\{\begin{array}{l}
\frac{\beta_{i}}{1-\left(1-\beta_{i}\right) \prod_{j=1, j \neq i}^{n}\left(1-\rho_{j} \beta_{j}\right)}, k=i \\
\frac{\rho_{k} \beta_{k}}{1-\left(1-\beta_{i}\right) \prod_{j=1, j \neq i}^{n}\left(1-\rho_{j} \beta_{j}\right)}, \text { else }
\end{array}\right.
$$


With Eq. (8), given the conditional collision probability $p_{c, k}$, the probability that node $k$ 's attempt is collision free seen by node $i$, denoted by $P_{t r}(k, i)$, is equal to:

$$
P_{t r}(k, i)= \begin{cases}P_{a}(i, i)\left(1-p_{c, i}\right), & k=i \\ P_{a}(k, i)\left(1-p_{c, k}\right) \frac{1-\beta_{i}}{1-\rho_{i} \beta_{i}}, & \text { else }\end{cases}
$$

When the channel is busy, the collision probability of the system seen by node $i$, denoted by $P_{\text {coll }}(i)$, is equal to:

$$
P_{\text {coll }}(i)=1-\sum_{k=1}^{n} P_{t r}(k, i)
$$

For the attempt process of node $i$, node $i$ 's attempts can be separated by renewal cycles. Each cycle consists an idle period followed by a busy period. The mean channel busy time seen by node $i$ before it obtains its transmission chance follows:

$$
E\left(t_{b u s y, i}\right)=\sum_{k=1}^{n} P_{t r}(k, i) \delta_{k}+P_{\text {coll }}(i) T_{c}
$$

where

$$
\delta_{k}=\left(1-p_{e, k}\right) t x\left(D A T A_{k}\right)+p_{e, k} \alpha_{k}
$$

$\alpha_{k}$ is defined by Eq. (44), and $T_{c}$ is the mean time the channel is sensed busy during a collision, and is equal to $t x(R T S)+E I F S$.

The mean number of renewal cycles between node $i$ 's consecutive attempts follows geometric distribution with parameter $P_{a}(i, i)$. Thus, the mean time period between node $i$ 's consecutive attempts is equal to $\frac{E\left(t_{\text {idle }, i}\right)+E\left(t_{\text {bus }}, i\right)}{P_{a}(i, i)}$. Considering collisions and channel errors, the mean number of attempts node $i$ needed for a successful transmission follows geometric distribution with parameter $1-\gamma_{i}$. Since each packet at node $i$ will be dropped after $K_{i}+1$ transmissions, the adjusted mean number of attempts for a packet follows: $\sum_{k=0}^{K_{i}}\left(1-\gamma_{i}\right) \gamma_{i}^{k}=\frac{1-\gamma_{i}^{K_{i}+1}}{1-\gamma_{i}}$. With Lhopital's rule for $\frac{0}{0}, \lim _{\gamma_{i} \rightarrow 1} \frac{1-\gamma_{i}^{K_{i}+1}}{1-\gamma_{i}}=K_{i}+1$. Therefore, the mean service time of node $i$ can be obtained by:

$$
E\left(S_{i}\right)=\frac{1-\gamma_{i}^{K_{i}+1}}{1-\gamma_{i}}\left(\frac{E\left(t_{\text {idle }, i}\right)+E\left(t_{\text {busy }, i}\right)}{P_{a}(i, i)}\right)
$$

We can use the service time to derive throughput. With queueing theory [13, the throughput of node $i$, denoted by $T_{i}$, follows:

$$
T_{i}=\frac{\rho_{i} D A T A_{i}\left(1-p_{d, i}\right)}{E\left(S_{i}\right) \delta}
$$

Where $\delta$ is the length of a time slot. $p_{d, i}$ reflects the impact of packet dropping, and follows:

$$
p_{d, i}=1-\sum_{k=0}^{K_{i}}\left(1-\gamma_{i}\right) \gamma_{i}^{k}=\gamma_{i}^{K_{i}+1}
$$




\subsection{The Existence of Equilibrium}

Let $\Gamma\left(p_{c, i}\right)=1-\prod_{j=1, j \neq i}^{n}\left(1-\rho_{j} G_{j}\left(p_{c, j}\right)\right)$, for $1 \leq i \leq n$, where $G_{i}\left(p_{c, i}\right)=\beta_{i}$. We can write these $n$ equations compactly in the following form of the simultaneous fixed point equations.

$$
\left(p_{c, 1}, p_{c, 2}, \ldots, p_{c, n}\right)=\left(\Gamma\left(p_{c, 1}\right), \Gamma\left(p_{c, 2}\right), \ldots, \Gamma\left(p_{c, n}\right)\right)
$$

Definition 1. Given $\boldsymbol{\rho}=\left(\rho_{1}, \ldots, \rho_{i}\right)$, the network is in equilibrium if the conditional collision probability vector $\boldsymbol{p}_{c}=\left(p_{c, 1}, \ldots, p_{c, n}\right)$ satisfies the simultaneous equations (19).

Because Eqs. (19) are non-linear equations, the network will be in multiple equilibria if the equations have multiple roots. In contrast, the network stays in a steady-state equilibrium if the equations have a unique root. The steady-state equilibrium can be directly used to describe the network's time average performance1, which is of fundamental interest.

Since $\boldsymbol{\rho}$ and $\boldsymbol{p}_{c}$ are continuous real vectors in $[0,1]^{n}$, Eqs.(19) are continuous function mapping from $[0,1]^{n}$ to $[0,1]^{n}$. Hence by Brouwer's fixed point theorem there exists fixed points in $[0,1]^{n}$ for Eqs. (19). Many existing algorithms can be used to calculate the roots of Eq. 19. For example, Quasi-Newton algorithms (e.g. the Broyden algorithm [14]) can be used to solve the equations efficiently. During our study, the equations can be solved quite fast and the root can be found with less than 7 iterations.

\subsection{Uniqueness of Equilibrium}

The network has a unique equilibrium if and only if Eqs.19) has a unique root. At the first glance, the uniqueness of solution may be affected by system parameters, BERs and the traffic arrival rate $\lambda$ s. Through detailed analysis, we can show that the uniqueness of equilibrium is only related to the settings of backoff parameters (i.e. $b_{i, k}$ ). In the following sub-sections, we give the details of analysis.

Interval Extensions of Rational Functions. We start our analysis by introducing basics of interval analysis. A real interval A is the bounded, closed set of the real numbers defined by

$$
A=[\underline{a}, \bar{a}]=\{x \in R \mid \underline{a} \leq x \leq \bar{a}\}
$$

where $\underline{a}, \bar{a} \in R$ and $\underline{a} \leq \bar{a}$. When $\underline{a}=\bar{a}$, A is called a singleton. An interval vector $\bar{V}$ in $I\left(R^{n}\right)$ has $n$ components, each of which is an interval, $V_{i} \in I(R)$, $i=1, \ldots, n$. For $A, B \in I(R)$, the operators $+,-, *, /$ are defined as follows:

$$
\begin{aligned}
A+B & =[\underline{a}+\underline{b}, \bar{a}+\bar{b}] \\
A-B & =[\underline{a}-\bar{b}, \bar{a}+\underline{b}] \\
A * B & =[\min (\underline{a b}, \underline{a} \bar{b}, \bar{a} \underline{b}, \bar{a} \bar{b}), \max (\underline{a b}, \underline{a} \bar{b}, \bar{a} \underline{b}, \bar{a} \bar{b})] \\
A / B & =A *[1 / \bar{b}, 1 / \underline{b}]
\end{aligned}
$$

${ }^{1}$ The time average is the long term time average, and does not necessarily prevent the short-term unfairness problem found in 8 . 
For an interval matrix A with interval coefficients $\left[\underline{a}_{i j}, \bar{a}_{i j}\right]$, its norm follows:

$$
\|A\|=\max _{i} \sum_{j=1}^{n} \max \left(\left|\underline{a}_{i j}\right|,\left|\bar{a}_{i j}\right|\right)
$$

As state in [15] that, given a rational function $f(x), x \in R^{n}$, its interval extension $F(X), X \subseteq I\left(R^{n}\right)$ can be obtained by simply replacing the real operations by interval operations and the variables by intervals. One simple example is,

$$
f(x)=x(1-x) ; F(X)=X *(1-X)
$$

Testing the Uniqueness of Solution. We apply the interval fixed point theorem to get the sufficient condition for the unique solution of Eqs.(19). In particular, given a initial $x_{0} \in X$, where $X$ is an interval vector. The Krawczyk operator [16] is defined as:

$$
\left.K\left(X, x_{0}\right)=x_{0}-Y f\left(x_{0}\right)+(I-Y J(X))\left(X-x_{0}\right)\right)
$$

where $Y$ is an arbitrary nonsingular matrix and $J(X)$ is the interval extension of the Jacobian of $F(x)$, which is denoted by $J(x)$. Specifically, $J_{i j}(X)$ is obtained by applying interval extension to $J_{i j}(x)$. Krawczyk [16] has shown that if $X$ has a solution to $F(x)=0$, where $F($.$) is a function, then so does K\left(X, x_{0}\right)$. According to the Moore's theorem [15, the sufficient condition for the uniqueness of solution for $F(x)=0$ is as follows:

$$
\|I-Y J(\mathbf{X})\|<1
$$

where $I$ is the identity matrix with the same dimension of $Y J(X)$. The norm of interval matrix $A$ with interval coefficients $A_{i j}=\left[\underline{a}_{i j}, \bar{a}_{i j}\right]$ is defined in Eqs. (21). The reason behind is that, when Eq.(22) holds, the Krawczyk operator performs contractive mapping in $X$ and guarantees a unique root.

In order to guarantee the uniqueness of solution, we need to find a nonsingular matrix $Y$ which satisfies condition (22). Supposed we have a nonsingular matrix $Y^{\prime}$, we relax condition (22) to reduce the computational complexity. Specifically,

$$
\left\|I-Y^{\prime} J(\mathbf{X})\right\|=\left\|Y^{\prime}\left(Y^{\prime-1}-J(X)\right)\right\|
$$

Since all matrix norms satisfy the submultiplicative property

$$
\|A B\| \leq\|A\|\|B\|
$$

Letting $Y=Y^{\prime-1}$, we have

$$
\left\|Y^{-1}(Y-J(X))\right\| \leq\left\|Y^{-1}\right\|\|Y-J(X)\|
$$

Since $\|I\|=1$, to satisfy $\left\|Y^{-1}||\right\| Y-J(X) \|<1$, we obtain

$$
\left\|Y ^ { - 1 } \left|\||| Y-J(X)\|<\left\|Y^{-1}|\|\mid Y\| \Rightarrow\|Y-J(X)\|<\|Y\|\right.\right.\right.
$$

Therefore, we need to study if we can find a nonsingular matrix $Y$ to satisfy Ineq. (23). 
The Jacobian $J(x)$ is calculated by:

$$
J_{i j}(x)= \begin{cases}-1, & i=j \\ \frac{\partial \Gamma\left(x_{i}\right)}{\partial x_{j}}, & \text { else }\end{cases}
$$

$\frac{\partial \Gamma\left(x_{i}\right)}{\partial x_{j}}$ is calculated as follows:

$$
\frac{\partial \Gamma\left(x_{i}\right)}{\partial x_{j}}=\prod_{k=1, k \neq i, j}^{n}\left(1-\rho_{k} G_{k}\left(x_{k}\right)\right) \rho_{j} G_{i}^{\prime}\left(x_{j}\right)
$$

Since $J_{i j}(x)$ is a rational function, we can easily apply interval extension to $J_{i j}(x)$ over the interval $[0,1]$. We have

$$
\begin{aligned}
& G([0,1])=[G(1), G(0)] \\
& \Rightarrow \prod_{k=1, k \neq i, j}^{n}\left(1-\rho_{k} G([0,1])=\right. \\
& {\left[\prod_{k=1, k \neq i, j}^{n}\left(1-\rho_{k} G(0)\right), \prod_{k=1, k \neq i, j}^{n}\left(1-\rho_{k} G(1)\right)\right]}
\end{aligned}
$$

Since $G_{i}($.$) resides in [0,1] and is a monotonically decreasing function [7] provided$ that $b_{i, k}$ is a non-decreasing sequence, $G_{i}^{\prime}()<$.0 . Thus, we have

$$
\begin{array}{r}
G_{i}^{\prime}([0,1])=\frac{\left(1-p_{e, i}\right) \rho_{i} \prod_{k=1, k \neq i, j}^{n}\left(1-\rho_{k} G_{i}(1)\right)}{\left(\sum_{k=0}^{K_{i}} b_{i, k} p_{e, i}^{k}\right)^{2}} * \\
{\left[\sum_{k=1}^{K_{i}} k b_{i, k} p_{e, i}^{k-1} \sum_{k=0}^{K_{i}} p_{e, i}^{k}-\frac{K_{i}\left(K_{i}+1\right)}{2} \sum_{k=0}^{K_{i}} b_{i, k}, 0\right]}
\end{array}
$$

Therefore, $J([0,1])$ follows:

$$
J_{i j}([0,1])=\left\{\begin{array}{l}
{[-1,-1], i=j} \\
{\left[\underline{a}_{i j}, \bar{a}_{i j}\right], \text { else }}
\end{array}\right.
$$

where

$$
\left[\underline{a}_{i j}, \bar{a}_{i j}\right]=G_{i}^{\prime}([0,1]) *\left[\prod_{k=1, k \neq i, j}^{n}\left(1-\rho_{k} G(0)\right), \prod_{k=1, k \neq i, j}^{n}\left(1-\rho_{k} G(1)\right)\right]
$$

Theorem 1. For each node $i$, if $b_{i, k}$ is non-decreasing, then the network has a unique equilibrium and the corresponding $\boldsymbol{\lambda}$ is also unique.

Proof. The existence of equilibrium has been proven in Section 3.3. Because all parameters are finite and $G($.$) is a non-increasing function, \left[\underline{a}_{i j}, \bar{a}_{i j}\right], 1 \leq i, j \leq n$ are compact and $\underline{a}_{i j}<\bar{a}_{i j}=0$. Given $J([0,1])$, we can always construct a nonsingular matrix $Y$ following the algorithm in Table 1. 
Table 1. The algorithm for constructing the matrix Y

$\mathrm{k}=\operatorname{argmax}_{1 \leq i \leq n} \sum_{j=1}^{n}\left|\underline{a}_{i j}\right| ; / *$ Note that $\underline{a}_{i}<0^{*} /$

Arbitrarily select a real positive number $\tau$, and matrix $Y$ is defined by:

$$
Y_{i j}= \begin{cases}-1, & i=j \\ \underline{a}_{k j}-\tau, & i=k \wedge i \neq j \\ 0, & \text { else }\end{cases}
$$

Applying the algorithm in Table 1 , it is obvious that $Y$ exists since $\left[\underline{a}_{i j}, \bar{a}_{i j}\right], 1$ $\leq i, j \leq n$ are compact. In addition, $Y$ is nonsingular since $\operatorname{det}(Y)=(-1)^{n}$. Applying $Y$ to the left-hand of Ineq (23), we have:

$$
\begin{aligned}
\|Y-J([0,1])\| & =(n-1) \sum_{j=1}^{n}\left|\underline{a}_{k j}-\tau\right| \\
& =\|Y\|-1<\|Y\|
\end{aligned}
$$

Therefore, Ineq. (23) is satisfied and, given $\boldsymbol{\rho}$, Eqs. (19) has a unique root $\boldsymbol{p}_{c}$. As a result, the network equilibrium is unique. In addition, according to the analysis in Section 3.2, the service time of node i, $E\left(S_{i}\right)$, is uniquely decided by $\rho$ and $\boldsymbol{p}_{c}$. Recalling Eq.(1), the mapping from $\boldsymbol{\rho}$ to $\boldsymbol{\lambda}$ is one-to-one, which guarantees the flow control vector $\boldsymbol{\lambda}$ is also unique.

\section{Model Validations}

We validate our model via comparing analytical results to simulation results. The simulations are performed using ns-2 17. Without loss of generalization, packet size is assumed to be 1000 bytes and the transmission rate of each node is assumed to be $2 \mathrm{Mbps}$ with a certain BER. Due to the limitation of space, we study a homogeneous network where each node follows the standard $802.11 \mathrm{~b}$

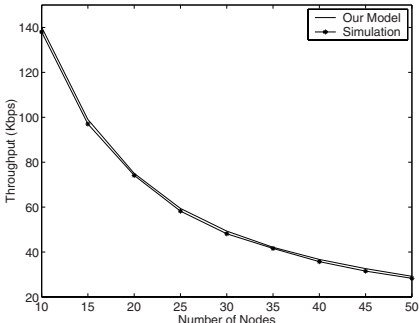

(a) Error-free Channel

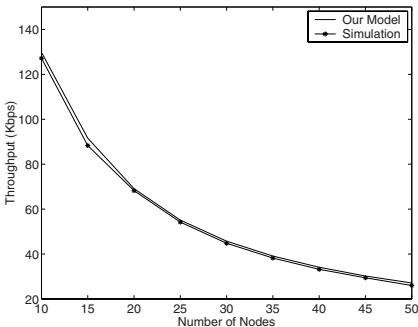

(b) Error-Prone Channel

Fig. 3. The average throughput 
specifications. In order to study both saturated and unsaturated traffic scenarios, $\rho_{i}$ is defined by $\min (0.05 * n, 1.0)$, where $n$ is the number of nodes.

We evaluate the average per-flow throughput in two scenarios, which are errorfree and error-prone respectively. When channel is error-prone, the BER of each flow is assumed to be equal to $10^{-5}$. We evaluate the average throughput as the function of the number of nodes. The results are shown in Figure 3 (a) and (b). As shown in the Figure, our model is able to accurately capture of average throughput in the system. Note that when the number of nodes is greater or equal to 20, every flow becomes saturated.

\section{Conclusion}

In this paper, we present a simple and accurate analysis model for the MAC performance of heterogeneous IEEE 802.11 based WLANs. We take into account of many practical factors (such as channel errors and unsaturated traffic) which may have significant impact on the network performance. With fixed point analysis, we first model the performance of the network with a simultaneous non-linear equations. Then, the service time of each flow is modeled and is further used to obtain the average throughput. We then theoretically examine the equilibrium of a general heterogeneous WLAN by studying the property of the corresponding simultaneous non-linear equations. We find the sufficient condition for the uniqueness of the equilibrium. Our model is validated through simulations and the results prove that our model is quite accurate to model the time average performance of the network. As future work, we will extend our model for 802.11-based multi-hop wireless ad hoc networks and study the existence and uniqueness of equilibrium as well.

\section{References}

1. IEEE: Wireless LAN Medium Access Control (MAC) and Physical Layer (PHY) Spec. IEEE 802.11 standard (1999)

2. 802.11 Work Group: Draft Supplement to Standard for Telecommunications and Information Exchange between Systems-LAN/MAN Specific Requirements- Part 11: Wireless LAN Medium Access Control (MAC) and Physical Layer (PHY) Specifications: Medium Access Control (MAC) Enhancements for Quality of Serice (QoS). IEEE 802.11e Draft 3.1 (2002)

3. Bianchi, G.: Performance analysis of the ieee 802.11 distributed coordination function. IEEE Journal on selected areas in communication 18(3) (2000) 535-547

4. Erge, M., Varaiya, P.: Throughput analysis and admission control for ieee 802.11a. Mobile Networks and Applications 10(5) (2005) 705-716

5. Garetto1, M., Chiasserini, C.F.: Performance analysis of 802.11 wlans under sporadic traffic. In: 4th International IFIP-TC6 Networking Conference (NETWORKING 2005). (2005)

6. Carvalho, M., Garcia-Luna-Aceves, J.: Delay analysis of ieee 802.11 in single-hop networks. In: IEEE ICNP'03. (2003)

7. Kumar, A., Altman, E., Miorandi, D., Goyal, M.: New insights from a fixed point analysis of single cell ieee 802.11 wlans. In: IEEE INFOCOM'05. (2005) 
8. Ramaiyan, V., Kumar, A., Altman, E.: Fixed pointed analysis of single cell ieee 802.11e wlans: Uniqueness, multistability and throughput differentiation. In: ACM SIGMETRICS'05. (2005)

9. Kim, H., Hou, J.C.: A fast simulation framework for ieee 802.11-operated wireless lans. In: ACM SIGMETRICS'04. (2004)

10. Medepalli, K., Tobagi, F.A.: Towards performance modeling of ieee 802.11 based wireless networks: A unified framework and its applications. In: IEEE INFOCOM'06. (2006)

11. Ross, S.M.: Introduction to Probability Models (7th Ed.). Academic Press (2000)

12. Mermaier, A.: Interval Methods for Systems of Equations. Cambridge University Press (1990)

13. Gross, D., Harris, C.M.: Fundamentals of Queueing Theory (Wiley Series in Probability and Statistics). Wiley-Interscience, 3rd edition (1998)

14. Broyden, C.G.: A class of methods for solving nonlinear simultaneous equations. Methematics of Computation 19 (1965) 577-593

15. Moore, R.E.: Interval Analysis. Prentice-Hall (1966)

16. Krawczyk, R.: Newton-algorithmen zur bestimmung von nullstellen mit fehlerschranken. Computing (by Springer Wien) 4(3) (1969) $187-201$

17. VINT Group: Network simulatior version 2 (ns-2). http://www.isi.edu/nsnam/ns/ (1999) 